Research Article

\title{
Prescribing pattern of proton pump inhibitors in the management of acid peptic disorders, focus on ilaprazole
}

\author{
Mayur M. Mayabhate*
}

\begin{abstract}
Medical Advisor, Medical
Science Division, Abbott India, Limited, Mumbai, Maharashtra, India
\end{abstract}

Received: 13 April 2016

Accepted: 07 May 2016

*Correspondence to:

Dr. Mayur M. Mayabhate,

Email: mayur.mayabhate

@abbott.com

Copyright: (C) the author(s), publisher and licensee Medip Academy. This is an openaccess article distributed under the terms of the Creative Commons Attribution NonCommercial License, which permits unrestricted noncommercial use, distribution, and reproduction in any medium, provided the original work is properly cited.

\begin{abstract}
Background: Acid peptic disorders (APDs) result from characteristic but overlapping pathogenic mechanisms that involve acid effects on diminished mucosal defense. Proton pump inhibitors (PPIs) have become a mainstay in the APDs. This survey was aimed to determine the usage profile of different proton pump inhibitors and overall physicians' opinion about the clinical experience with newer PPI, ilaprazole.

Methods: This survey was based on the clinical experience of 107 randomly selected physicians on an aggregate patient basis in the management of acidpeptic disorders from different parts of India. A data report form (DRF) comprising of various questions was answered by these physicians. The responding physicians answered the DRF of patients who suffered from acidpeptic disorders and were eligible for PPI therapy. A minimum of 50 patients per physician were evaluated in this survey.

Results: The commonly encountered APD in physicians' clinical practice were gastroesophageal reflux disorder (GERD) followed by non-ulcer disease. $58.88 \%$ physicians highlighted the prevalence of night time symptoms of APD reported by the patients. About 1/4th physicians were of the opinion that longacting PPIs would be the preferred to offer 24 hour control of gastric acid secretion thereby controlling the nocturnal symptoms. $63.73 \%$ of physicians reported significantly better symptomatic relief with once daily dose of ilaprazole than existing PPIs.

Conclusions: This survey highlights the lack of 24 hour acid control in APDs with use of conventional PPIs leading to variety of symptoms, especially at night, seriously hampering the sleep quality. Ilaprazole displays important advantages in the clinical settings, with regard to better probability of extending the inhibition of acid secretion over 24 hours as compared to conventional PPIs.
\end{abstract}

Keywords: APDs, 24 Hour acid control, Ilaprazole

\section{INTRODUCTION}

Acid peptic disorders (APDs) are a collective term that includes many conditions such as peptic ulcer disease, GERD and Gastritis. Acid peptic disorders are the result of distinctive, but overlapping pathogenic mechanisms leading to either excessive acid secretion or diminished mucosal defense. ${ }^{1}$

The prevalence of gastroesophageal reflux disease (GERD) in India is likely to be between $8 \%$ and $19 \%$, which is comparable to GERD prevalence rates published in western countries. ${ }^{2}$ Peptic ulcer disease (PUD) tends to have a chronic remitting course with imperfect correlation between symptoms and the presence of an ulcer. Each year PUD affects 4 million people around the world. A recent study by Dutta AK et al showed a time trends in epidemiology of peptic ulcer disease in India and observed the frequencies of $2.9 \%$ for duodenal ulcer and $2.7 \%$ gastric ulcer from 1988 to $2008 .^{3}$

The causes of APD include H. Pylori infection, over use of NSAIDS, smoking and alcohol consumption and it is also linked with stress and dietary factors such as spicy and oily food. The increase in incidence of APD in the current scenario can be linked to the present stressful lifestyles. Patients commonly present with nausea, heartburn, dysphagia and abdominal pain. If left 
uncontrolled, it may lead to complications such as ulceration, perforation, strictures, gastric outlet obstruction and even malignancy. ${ }^{2}$

Agents which can be used in the treatment of APD include antacids, acid reducing drugs and mucosal protective agents. Currently, therapy is essentially aimed at decreasing gastric acid and the most common drugs used are $\mathrm{H}_{2}$ antagonists and proton pump inhibitors (PPIs). ${ }^{4,5}$

Currently, PPIs (omeprazole, lansoprazole, pantoprazole, rabeprazole and esomeprazole) are widely used for the treatment of APDs and have short half-lives which range from 1 to 1.5 hours. All 5 PPIs are effective and safe; however, there are differences in PPI pharmacokinetic and pharmacodynamics profiles that might influence their clinical utility. ${ }^{5}$ The lately introduced novel proton pump inhibitor ilaprazole (2-[(4methoxy-3-methyl)-2-pyridinyl] methylsulfinyl-5-(1H-pyrrol-1yl)-1H-benzimidazole) is a substituted benzimidazole derivative. The prolonged inhibitory effect of ilaprazole is attributed to its longer plasma half-life $\left(\mathrm{t}_{1 / 2}=3.6\right.$ hours $)$ compared with other PPIs, which increases the exposure of the $\mathrm{H}^{+} / \mathrm{K}^{+}$-ATPase pumps to the drug. Ilaprazole might thus offer potential pharmacological advantages over omeprazole and, possibly, other, similar PPIs. ${ }^{6}$ A PPI with longer half-life would produce a more prolonged blockade of proton pumps with the potential for greater acid suppression and hence, greater clinical effect, particularly for patients with postprandial evening and/or nocturnal symptoms. ${ }^{7}$ The nocturnal symptoms of APD, including heartburn, a bitter or sour taste in the mouth, a burning sensation in throat, not only adversely affect sleep, but also impair functional abilities during the day. ${ }^{8}$

The objective of this survey was to determine the usage profile of different proton pump inhibitors and an overall physicians' opinion about the clinical experience with ilaprazole as per approved label indications.

\section{METHODS}

This survey was based on the clinical experience of 107 randomly selected physicians (gastroenterologists, gastro surgeons, consulting physicians and general practitioners) on an aggregate patient basis in the management of acidpeptic disorders from different parts of India. A data report form (DRF) comprising of various questions was answered by these physicians. The responding physicians answered the DRF of patients who suffered from acidpeptic disorders and were eligible for PPI therapy. A minimum of 50 patients per physician were evaluated in this survey. The key elements of the questionnaire were as follows: commonly encountered acid peptic disorders in clinical practice (such as GERD, peptic ulcer disease, non-ulcer disease etc.), conditions commonly present with symptoms (such as epigastric pain, heartburn, bloating, regurgitation, sleep disturbances etc.), commonly used PPIs/ $\mathrm{H}_{2} \mathrm{RAs}$ for management of APDs, percentage of patients having nocturnal symptoms, percentage of patients having trouble sleeping, preferred management strategy for nocturnal symptoms of APD (doubling dose of PPI, long-acting PPIs, add antacids $\mathrm{H}_{2}$ receptor antagonist), perceived symptomatic relief with ilaprazole and lastly the potential candidates for longer acting PPIs. An approval from the ethics committee was not required as this survey involved only the physicians' opinion on the use of PPIs in their clinical practice and did not involve the direct participation of any patient.

\section{Stastistical analysis}

Data entry was done in Microsoft excel 2013 (version office 365) in a study (questionnaire) specific excel spread sheet validated for the 10 item study questionnaire. Validation was done using built-in validation tools in MS excel to minimize data entry errors.

Data for responses to multiple questions were expressed as numbers with percentages (\%) for each response. Percentage values were calculated based on the total number of responders for each question independently.

\section{RESULTS}

The study questionnaire was sent to 107 participating doctors spread across India. The results were based on the percentage value of the number of individually answered questions.

In the present survey it was seen that GERD was most common $(42.57 \%)$ APD, followed by non-ulcer disease $(32.28 \%)$ and peptic ulcer disease $(19.07 \%)$. The patients most commonly presented with heart burn, epigastric pain, bloating, regurgitation, nausea, vomiting and sleep disturbances. PPIs are the most preferred drugs for management of APDs. PPIs were most frequently prescribed in the mornings before breakfast, followed by twice daily before meals in certain cases.

Patients often experienced symptoms of APDs, especially during night. $58.88 \%$ physicians reported night time symptoms of APD by the patients while $29.91 \%$ physicians reported the same in the evening hours. Upto $80 \%$ of physicians feel that about $30 \%$ patients of APD do report experience symptoms at night. Because of this suffering, $61 \%$ of physicians feel that these patients have reported the sleep disturbances one to twice or even more per week (Figure 1).

Physicians mostly doubled the dose of PPI if patient experienced nocturnal symptoms, however, one fourth of physicians feel that these patients are the potential candidates for longer half-life PPI (23.9\%).

As far as ilaprazole is concerned, $63.73 \%$ of physicians feel significantly better symptomatic relief on once daily dose of ilaprazole than on existing PPI, while $34.31 \%$ 
physicians reported symptomatic relief comparable to conventional PPI (Figure 2).

$47 \%$ physicians feel that, almost half of the patients of APD could be the potential candidates for longer acting PPIs for management of APD.

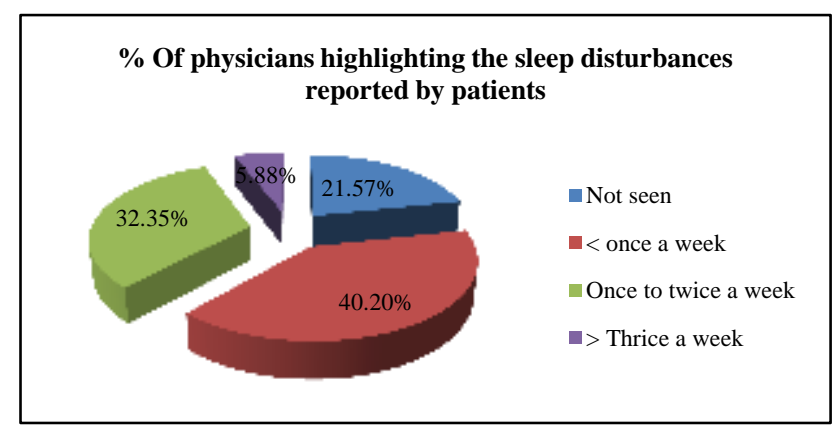

Figure 1: Frequency of sleep disturbances in APD highlighted by physicians.

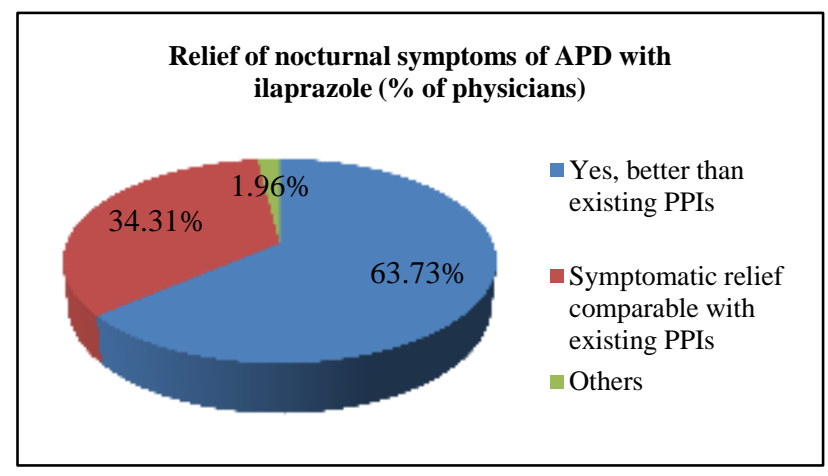

Figure 2: Relief of nocturnal symptoms with ilaprazole.

\section{DISCUSSION}

The proton pump inhibitors (PPIs) have become the standard of care in patients with acid peptic disorders. However, there still remain unmet needs in the pharmacological control of acid secretion. The major short coming relates to the lack of 24 hours control of acid secretion, the problems of nocturnal symptoms hampering quality of sleep and rebound acid hyper secretion with administration of PPIs. ${ }^{9}$ The present study showed that $58.88 \%$ of patients on conventional PPIs had breakthrough manifestations particularly at night, which is in line with reported literature. ${ }^{10}$

Of note, the favourable pharmacokinetic pattern of ilaprazole, relying mainly in a lack of influence by $\mathrm{CYP}_{2} \mathrm{C}_{19}$ genetic polymorphism and a prolonged elimination half-life, is expected to impact positively, in terms of lower variability and long lasting acid inhibition, on the inhibition of gastric acid secretion. ${ }^{11}$

In this survey, the commonly encountered acid peptic disorders observed were GERD followed by peptic ulcer disease. Commonly reported symptoms of APD are heartburn, regurgitation, abdominal discomfort, nausea, sleep disturbances. Our findings are in line with a recent study by Singh VK et al. and their colleagues, where they have collected the data from both physicians and patients to know prescribing pattern of proton pump Inhibitors and observed that GERD was the commonest indication for prescribing acid suppressant drugs by physicians and majority of physicians prescribed acid suppressants drugs for 1-3 months. ${ }^{12}$

About $1 / 4^{\text {th }}$ physicians were of the opinion that longacting PPIs would be the preferred treatment to offer 24 hour control of gastric acid secretion (especially during night time) and nocturnal symptoms.

According to the survey, $63.73 \%$ of physicians reported significantly better symptomatic relief on once daily dose of ilaprazole than on existing PPI, while $34.31 \%$ physicians reported symptomatic relief comparable to conventional PPI. This reflects the translation of pharmacokinetic superiority of ilaprazole into clinical practice as compared to conventional PPIs.

These findings are in line with recently published literature by Shin et al highlighting that ilaprazole works over 24 hours and offer better acid control as compared to esomeprazole especially in evening and overnight hours. ${ }^{13}$ Thus, many physicians from this study also opine that almost half of the patients of APD could be the potential candidates for longer acting PPIs for better symptomatic control. Expert opinion by Bartoli et al highlights that, ilaprazole $(10 \mathrm{mg})$ is better than conventional PPIs like pantoprazole, rabeprazole etc. in maintaining higher mean intragastric $\mathrm{pH}$ on day 5. About $70 \%$ of the times, ilaprazole maintained intragastric $\mathrm{pH}>4$, with mean $\mathrm{pH}$ of 4.8 on day 511. Thus, ilaprazole, a PPI with longer half-life, seems to be beneficial in management of APDs with superior $\mathrm{pH}$ control offering prompt symptomatic relief.

\section{CONCLUSION}

Physicians feel that lack of 24 hour acid control leads to symptoms of APD seriously hampering the sleep quality and consequently the quality of life. They opine that ilaprazole displays important advantages in their clinical practice, with regard to better probability of extending the inhibition of acid secretion over 24 hours as compared to conventional PPIs.

\section{Funding: Abbott India Ltd}

Conflict of interest: This survey and scientific publication was conceptualized, edited, designed and funded by Abbott India Ltd. Please consult full prescribing information before prescribing any of these products mentioned in this publication

Ethical approval: Not required 


\section{REFERENCES}

1. Alex M, Kraft WK. Acid peptic diseases: pharmacological approach to treatment. Expert Rev Clin Pharmacol. 2009;2(3):295-314.

2. Goodman L, Brunton L, Chabner B, Knollmann B. Goodman and Gilman's pharmacological basis of therapeutics. New York: McGraw-Hill; 2013.

3. Dutta AK, Chacko A, Balekuduru A, Sahu MK, Gangadharan SK. Time trends in epidemiology of peptic ulcer disease in India over two decades. Indian J Gastroenterol. 2012;31(3):111-5.

4. Gaddam S, Sharma P. Shedding light on the epidemiology of gastroesophageal reflux disease in India - a big step forward. Indian J Gastroenterol. 2011;30(3):105-7.

5. Robinson M, Horn J. Clinical pharmacology of proton pump inhibitors: what the practicing physician needs to know. Drugs. 2003;63(24):2739-54.

6. Goldwater R, Lee SM, Chung GJ, Kim DY, Cho $\mathrm{KD}$, Boileau F. A phase I, randomized, parallel placebo-controlled safety, tolerance and pharmacokinetic study of multiple doses of IY-8119 in fasting male volunteers. Clinical Pharmacology and Therapeutics. 1999;65(2):126.
7. Ang TL, Fock KM. Nocturnal acid breakthrough: clinical significance and management. J Gastroenterol Hepato. 2006:21(5);125-8.

8. Fass R. Nocturnal acid breakthrough: A critical assessment. Hospital Physician. 2004:47-52

9. Tytgat GNJ. Are there unmet needs in acid suppression? Best Practice and Research Clinical Gastroenterology. 2004;18:67-72.

10. Triantafillidis JK, Mylonaki M, Georgopoulos F. Nocturnal acid breakthrough: consequences and confronting. Annals of Gastroenterology. 2003;16:280-6.

11. Bortoli D, Martinucci I, Giacchino M, Blandizzi C, Marchi S, Savarino V, et al. The pharmacokinetics of ilaprazole for gastro-esophageal reflux treatment. Expert Opin Drug Metab Toxicol. 2013.9(10):1361-9.

12. Singh VK, Prabhu K, Ponnudurai K, Singh PK. Prescribing pattern of acid suppressants in modern clinical practice - An analysis. Der Pharmacia Sinica. 2011;2(3):67-73.

13. Shin JS, Lee JY, Cho KH, Park HL, Kukulka M, Wu $\mathrm{J}$, et al. The pharmacokinetics, pharmacodynamics and safety of oral doses of ilaprazole 10, 20 and 40 $\mathrm{mg}$ and esomeprazole $40 \mathrm{mg}$ in healthy subjects: a randomized, open-label crossover study. Aliment Pharmacol Ther. 2014;40(5):548-61.

Cite this article as: Mayabhate MM.

Prescribing pattern of PPIs in the management of acid peptic disorders, focus on ilaprazole. Int J Basic Clin Pharmacol 2016;5:1104-7. 